\title{
Localisation of malignant glioma by a radiolabelled human monoclonal antibody
}

\author{
JACK PHILLIPS, TOM ALDERSON, KAROL SIKORA, JAMES WATSON
}

From the Department of Neurosurgery, St. Laurence's Hospital, Dublin, the Ludwig Institute for Cancer

Research, Addenbrooke's Hospital, Cambridge, the Huntingdon Oncology Clinic, Hinchingbrooke Hospital, Huntingdon and the MRC Clinical Oncology Unit, Cambridge

SUMMARY Human monoclonal antibodies were produced by fusing intratumoral lymphocytes from patients with malignant gliomas with a human myeloma line. One antibody was selected for further study after screening for binding activity to glioma cell lines. The patient from whom it was derived developed recurrent glioma. $1 \mathrm{mg}$ of antibody was purified, radiolabelled with ${ }^{131} \mathrm{I}$, and administered intravenously. The distribution of antibody was determined in the blood, CSF and tumour cyst fluid and compared with that of a control human monoclonal immunoglobulin. Antibody localisation in the tumour was observed and confirmed by external scintiscanning. .

The current prognosis for patients with poorly differentiated malignant gliomas is extremely poor. Conventional treatment involves surgery followed by radiotherapy, and in some certres chemotherapy. ${ }^{1}$ Local recurrence occurs within months of initial therapy and is not usually amenable to further treatment.

There have been several reports describing the use of monoclonal antibodies to treat a variety of tumour types including leukaemia, ${ }^{2}$ lymphoma ${ }^{3}$ and colorectal cancer. ${ }^{4}$ Malignant gliomas are often heavily invaded by lymphocytes, presumably in response to antigens present on the tumour cell surface. ${ }^{5}$ In previous studies we have fused intratumoral lymphocytes from patients with glioma to a human myeloma line to obtain human hybridomas. ${ }^{6}$ These cells produce cloned immunoglobulins, some of which have tumour binding activity. Before the therapeutic exploitation of such antibodies can occur it is essential to determine their specificity in a range of tumours and normal tissues in vitro and also to determine whether such antibodies will localise tumour in vivo in patients with recurrent glioma. In this paper we describe the use of a human anti-glioma monoclonal antibody derived from a patient at time of surgery for primary disease to localise a recurrent cystic glioma in the same patient six months subsequently.

Address for reprint requests: Dr K Sikora, the Ludwig Institute for Cancer Research, The Medical School, Hills Road, Cambridge CB2 2QH. UK.

Received 14 September 1982. Accepted 20 October 1982

\section{Patient and methods}

The patient was a 21-year-old girl presenting with a six month history of headaches of increasing severity, together with progressive dementia. Investigations revealed a space occupying lesion in the right frontotemporal region and at craniotomy a large, grade III-IV astrocytoma was found in this area. Sub-total excision was performed and one gram of biopsy material placed in tissue culture medium. The tumour tissue was teased apart in sterile tissue culture medium consisting of Dulbecco's Modified Eagles Medium and $10 \%$ Foetal Calf Serum, penicillin $100 \mathrm{IU} / \mathrm{ml}$ and streptomycin $100 \mathrm{mg} / \mathrm{ml}$. The cell suspension was layered onto Ficoll Hypaque and centrifuged at room temperature for 30 minutes at $400 \mathrm{~g}$. Cells collecting at the interface were harvested and washed three times in medium. These glioma lymphocytes were then fused with an 8-azaguanine resistant human myeloma cell line, LICR-LON-HMy $2,{ }^{7}$ which dies on a hypoxanthine, aminopterin and thymidine (HAT) medium following a standard protocol. ${ }^{8}$ Hybrid clones were observed four weeks later (fig 1) and the supernatant tested in a solid phase radiommunoassay for binding to a rapidly growing human glioma line, GCCM. Binding activity was noted and one hybridoma clone LGL1-1D6 selected for further study.

Ten litres of hybridoma supernatant was obtained by growing the LGL1-1D6 line in roller bottles in Iscove's (Flow Laboratories) serum-free medium. ${ }^{9}$ The supernatant was precipitated with $50 \%$ ammonium sulphate and purified on a DE52 (Whatman) ion exchange column. $1 \mathrm{mg}$ of purified antibody was iodinated with ${ }^{131}$ I (Amersham) using a modification of the chloramine-T method. The iodinated antibody ( $1 \mathrm{mCi} / \mathrm{mg}$ protein) was filtered through a 22 micrometer Millex filter to ensure sterility and stored in 5\% human serum at $4^{\circ} \mathrm{C}$ prior to use. Autoradiography of a polyacrylamide gel which separates molecules on the basis of 


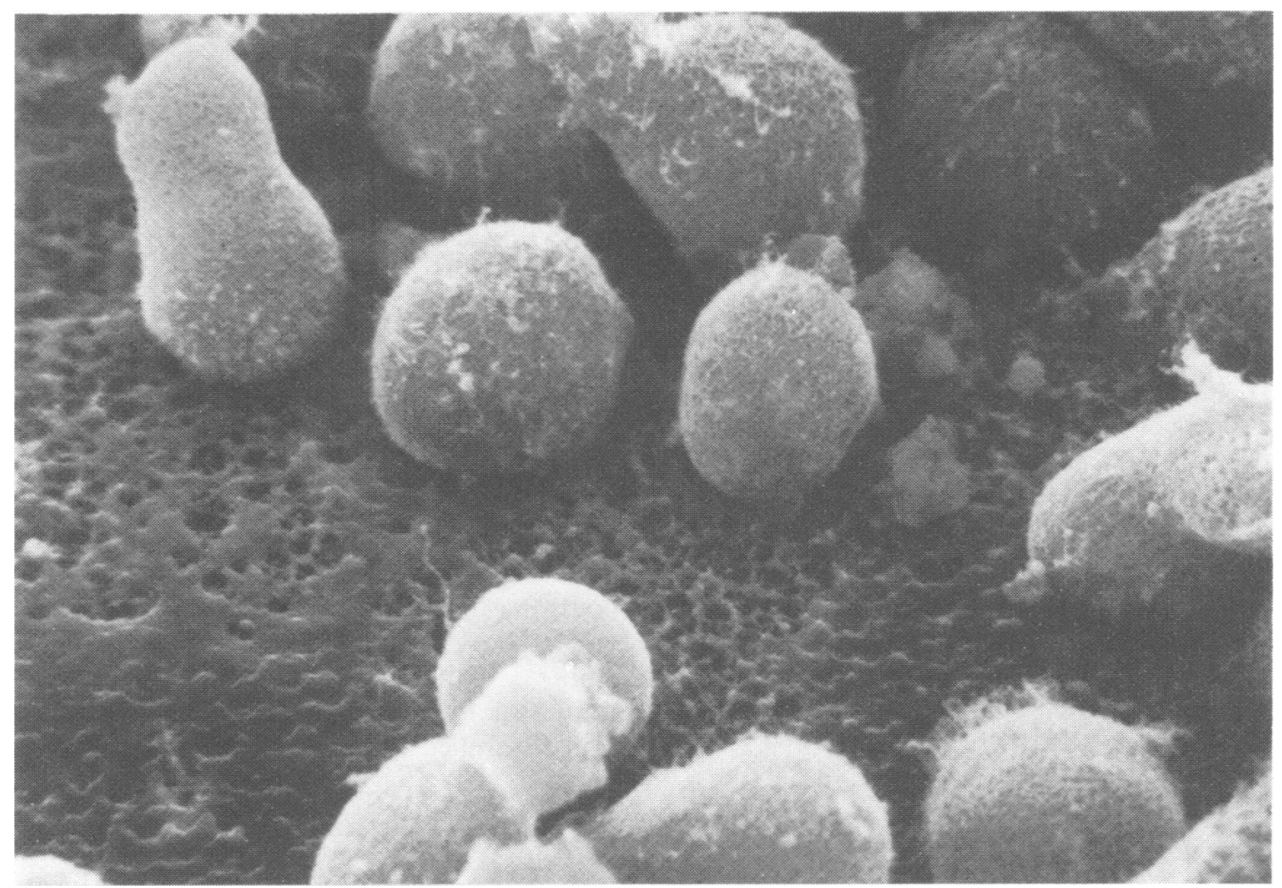

Fig 2 Autoradiography of SDS-polyacrylamide gel electrophoresis of ${ }^{131}$ I-labelled LGL1-1D6 monoclonal immunoglobulin.

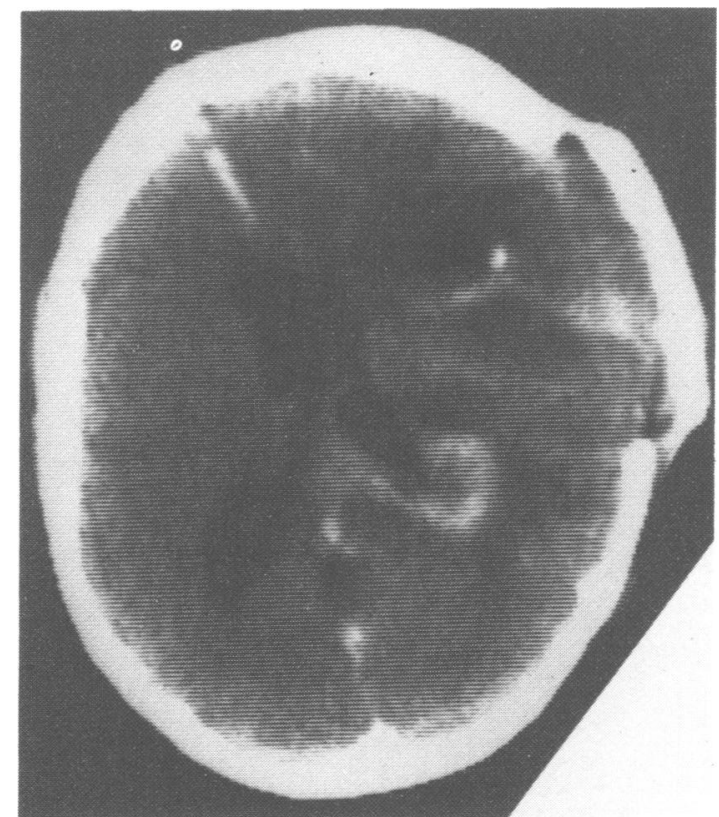

Fig 3 CT scan showing recurrent cystic glioma in right frontoparietal region. 
size was performed to check the purity of the final preparation (fig 2).

Six months following primary excision the patient's presenting symptoms returned and investigation revealed recurrent cystic tumour in the primary site (fig 3). A decompression operation was carried out. Following further cystic recurrence, with hydrocephalus and headache, Rickham reservoirs were placed beneath the scalp for aspiration of CSF and intratumoral fluid.

Following full discussion, ethical approval was obtained from the St Laurence's Hospital Ethical Committee and informed consent obtained from the patient's relatives to attempt tumour localisation with the purified human antibody. $1 \mathrm{mg}$ of labelled antibody was given by slow intravenous injection in $20 \mathrm{ml}$ normal saline into the ante-cubital vein. Serial samples of blood, CSF and tumour cyst fluid were removed daily following this injection. Brain scan images were obtained using a rectilinear scanner which recorded the total counts emitted and the ${ }^{131}$ I distribution. Three weeks subsequent to this $1 \mathrm{mg}$ of purified immunoglobulin from the parent human myeloma line LON-LICR-HMy2 similarly purified and labelled was injected intravenously and the studies repeated. This protein did not have antiglioma binding activity and was used as a control.
Results

No untoward side effects were noted after antibody administration. There were no changes in the patient's pulse, temperature, blood pressure, neurological signs or haematological indices. Figure 4 shows the radioactive counts in $100 \mu$ l of serum, CSF and tumour cyst fluid following administration of monoclonal antibody and control immunoglobulin. There was no significant difference between counts in the serum occurring after the administration of labelled monoclonal antibody or labelled controlled myeloma protein. Similarly there was no difference in the CSF counts. However, the ${ }^{131}$ I labelled monoclonal antibody persisted within the tumour cyst fluid for over six days. The amount of activity was five times higher than that of the control protein. External counting on either the left or right side of the cranial cavity was performed, as well as over the heart (table). Uptake was greater over the right frontotemporal area when compared to the left. There was no difference in the counts over the heart. A

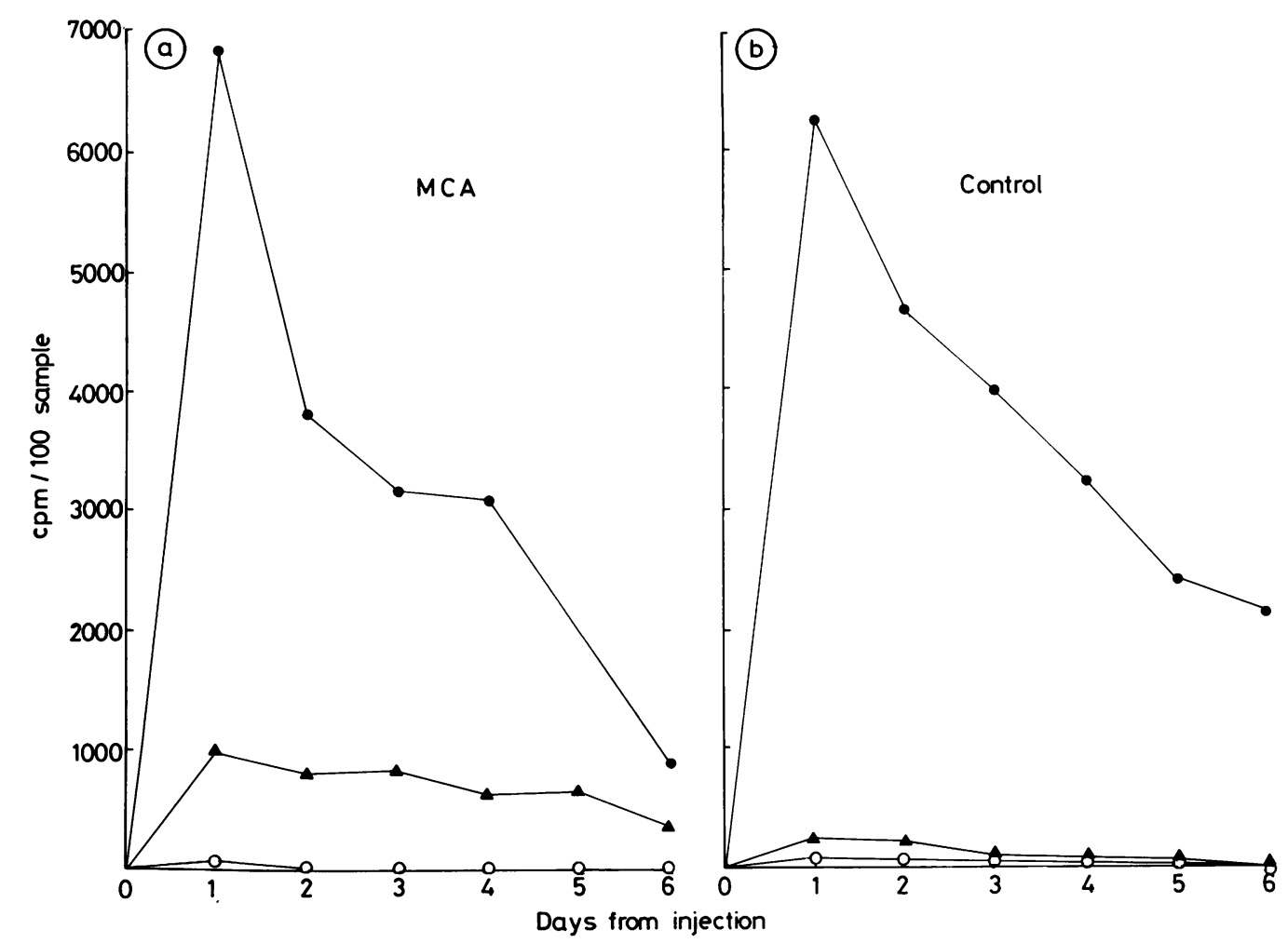

Fig $4{ }^{131}$ I-activity in serum (- - $)$ ); CSF $(\mathrm{O}-\mathrm{O})$ and tumour cyst fluid $(\mathbf{\Delta}-\mathbf{\Delta})$ after i.v. injection of labelled $1 \mathrm{D} 6$ monoclonal antibody or control monoclonal $\mathrm{IgG}$. 


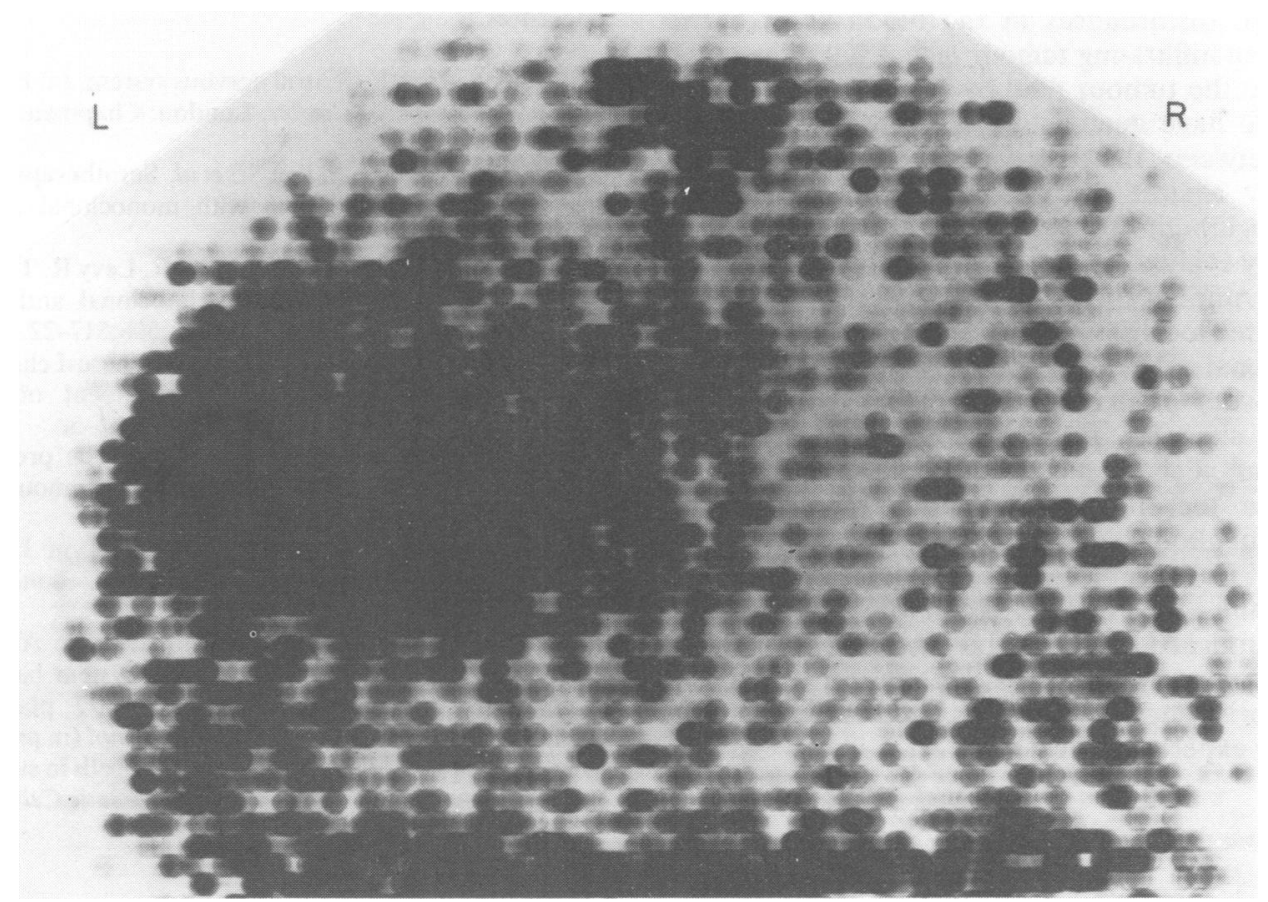

Fig 5 Rectilinear scan using ${ }^{131}$ I-1D6 monoclonal antibody frontal view.

rectilinear scan performed on day four after the monoclonal antibody administration revealed tumour localisation (fig 5). A Sephacryl 200 column was used to determine the molecular weight of radioactively labelled material within the tumour cyst fluid (fig 6). Although a small amount of free iodine

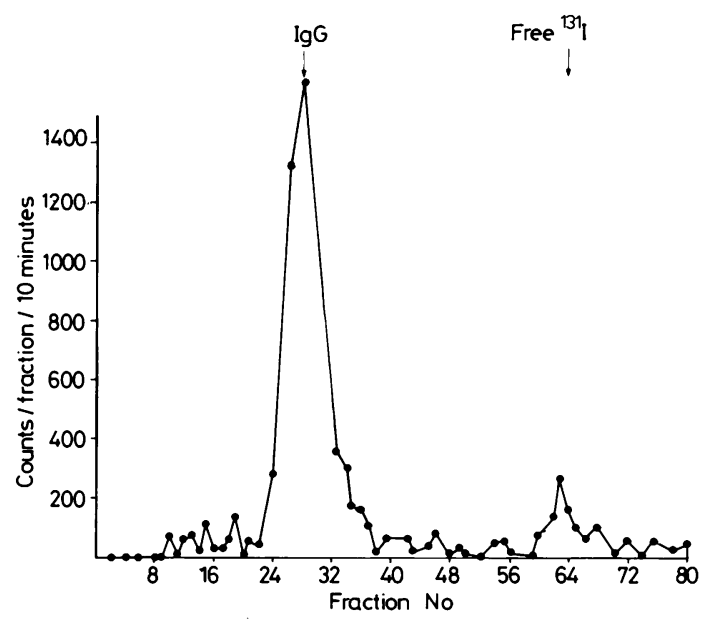

Fig 6 Analysis of distribution of ${ }^{131}$ I in tumour cyst fluid on sephacryl-200 exclusion column.
Table

\begin{tabular}{|c|c|c|c|c|c|c|}
\hline \multirow[t]{2}{*}{ Day of study } & \multicolumn{2}{|c|}{ (R) Brain } & \multicolumn{2}{|c|}{ (L) Brain } & \multicolumn{2}{|l|}{ Heart } \\
\hline & $M C A$ & $C O N$ & $M C A$ & CON & $M C A$ & CON \\
\hline 5 & 1300 & 800 & 400 & 400 & 2800 & 2300 \\
\hline 6 & 1100 & 600 & 300 & 200 & 1800 & 1600 \\
\hline 7 & 800 & 600 & 200 & 200 & 1800 & 1800 \\
\hline 8 & 700 & & 180 & & 700 & \\
\hline 10 & 500 & & 125 & & 400 & \\
\hline
\end{tabular}

was present, most of the radioactivity was in the region of the molecular weight of the monoclonal immunoglobulin, indicating that intact antibody molecules were present in the tumour cyst fluid.

\section{Discussion}

We have demonstrated previously that B-lymphocytes from malignant gliomas can be immortalised and cloned for further study. The immunoglobulins produced by these cells represent those released in the area of the tumour and may well be involved in host defence against the tumour. In this paper we have shown that radioactively labelled monoclonal antibody derived in this way can localise recurrent glioma in the patient from which it was derived. 
Although disturbances in the blood brain barrier around an infiltrating tumour may result in increased access to the tumour fluid by a range of serum proteins, we have demonstrated a considerable difference between the behaviour of the monoclonal antibody against the glioma and the monoclonal immunoglobulin from the parent myeloma. We are currently evaluating potential uses of such antibodies for determining spread of tumour prior to therapy. Adequate localisation is clearly essential if the monoclonal antibody technology is to be utilised for the delivery of drugs, toxins or radionucleides to tumour sites for therapeutic purposes. There is particular need to pursue this technology if it can influence the hopeless prognosis associated with malignant glioma.

We thank the nursing staff of the Hardwick Neurosurgical Unit at St Laurence's Hospital, the Royal College of Surgeons, Dublin, for use of their laboratories and Mr John Ellis and Ms Berni Horner for their expert technical help.

\section{References}

1 Jones A, Currie J. Central nervous system. In: Halnan K, ed. Treatment of Cancer. London: Chapman and Hall, 1982.

${ }^{2}$ Ritz J, Pesando JM, Sallan SE et al. Serotherapy of acute lymphoblastic leukemia with monoclonal antibody. Blood 1981;58:361-72.

${ }^{3}$ Miller RA, Maloney DG, Warnke R, Levy R. Treatment of B-cell lymphoma with monoclonal anti-idiotype antibodies. New Eng J Med 1982;306:517-22.

${ }^{4}$ Sears HF, Atkinson B, Mattis J et al. Phase-I clinical trial of monoclonal antibody in treatment of gastrointestinal tumours. Lancet 1982;I:762-5.

${ }^{5}$ Phillips JP, Eremin O, Anderson J. The presence of lymphoreticular cells in human brain tumours and in normal brain. BrJ Cancer (in press).

${ }^{6}$ Sikora K, Alderson T, Phillips J, Watson J. Human hybridomas from malignant gliomas. Lancet 1982; I:11-4.

${ }^{7}$ Edwards PAW, Smith CM, Munro Neville A, O'Hare MJ. A human-human hybridoma system based on a fast-growing mutant of the ARH-77 plasma cell leukaemia-derived line. Eur J Immunol (in press).

${ }^{8} \mathrm{Hales} A$. A procedure for the fusion of cells in suspension by means of polyethylene glycol. Somatic Cell Genetics 1977;3:227-35. 